\title{
Urban Regional Logistics Distribution Path Planning Considering Road Characteristics
}

\author{
Chuanxiang Ren $\left(\mathbb{D},{ }^{1}\right.$ Xiaoqi Wang $\mathbb{D}^{1},{ }^{1}$ Ge Gao $\mathbb{D}^{1},{ }^{1}$ and Juntao $\mathrm{Li}^{2}$ \\ ${ }^{1}$ College of Transportation, Shandong University of Science and Technology, Qingdao, Shandong Province 266590, China \\ ${ }^{2}$ Beijing Wuzi University, No. 321, Fuhe Street, Tongzhou District, Beijing 101100, China \\ Correspondence should be addressed to Chuanxiang Ren; renchx@sdust.edu.cn
}

Received 19 January 2020; Revised 11 April 2020; Accepted 9 May 2020; Published 1 June 2020

Academic Editor: Manuel De la Sen

Copyright (C) 2020 Chuanxiang Ren et al. This is an open access article distributed under the Creative Commons Attribution License, which permits unrestricted use, distribution, and reproduction in any medium, provided the original work is properly cited.

\begin{abstract}
Generally, road characteristics (such as the longitudinal slope and pavement damage) have an important effect on logistics distribution path, not only the vehicle fuel economy and driving safety but also the benefits and efficiency of logistics companies. It is necessary to explore the influence of road characteristics on logistics distribution path planning. First, a road characteristics evaluation value is defined to quantify road characteristics. With the application of analytic hierarchy process (AHP), the road characteristics evaluation index system with three criteria and eleven indices was built, and then the calculation of the road characteristics evaluation value is proposed based on the fuzzy comprehensive evaluation method. Secondly, a mathematical model of logistics distribution path planning is proposed, in which road characteristics and distance are comprehensively considered. Thirdly, an adaptive genetic algorithm (AGA) is presented with customized crossover operator for the solution of the mathematical model. Finally, through simulation by a real example, the influences of road characteristics and distance on the optimal distribution path are discussed, and the results show the model considering the road characteristics and distance comprehensively achieves superior distribution paths to that considering the distance or road characteristics only.
\end{abstract}

\section{Introduction}

With the rapid development of e-commerce and mobile Internet technology, the online shopping demands and capabilities of customers are getting higher, which brings the rapid development of urban regional logistics distribution services. In some big cities, such as Qingdao and Shanghai in China, almost every community has a small distribution station. Logistics distribution companies generally set up a distribution center in a certain area of the city, which covers several or dozens of communities. The logistics company deploys vehicles to deliver the goods received by the distribution center to distribution stations in each community every day. In China, it has become a common phenomenon and daily part of people's lives that goods at distribution stations are usually picked up by users themselves. This kind of urban regional logistics distribution, in which the vehicles assigned from distribution center to deliver goods to each distribution station every day, is becoming a common logistics distribution mode, and the schematic diagram of the logistics distribution process is shown in Figure 1.

In Figure 1, one distribution center, nine distribution stations, and three distribution paths (or vehicle paths) are illustrated. Nine distribution stations correspond to nine communities, and three of which with user paths are shown in detail. According to the planned distribution path, every vehicle starts from the distribution center, completes the distribution of goods at the distribution stations, and finally returns to the distribution center. In each community, users come to the distribution station to pick-up their own items based on their paths. During the logistics distribution process, the distribution path planning is an important part, which affects the benefits and efficiency of logistics companies [1-3]. The distribution path planning is related to the location relationship between distribution center and distribution stations, the demand of distribution stations, etc., 


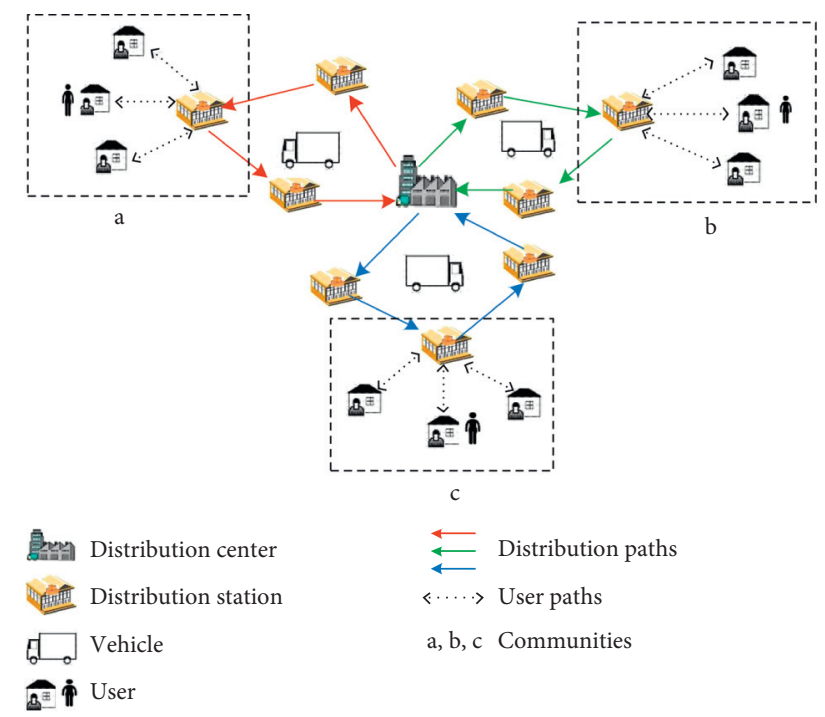

Figure 1: The schematic diagram of the urban regional logistics distribution process.

which belongs to a vehicle routing problem (VRP). In logistics distribution path planning, the road distance is often chosen as an important factor to evaluate the quality of the distribution path, such as establishing the path planning model with the minimum distance of all distribution paths as the goal to obtain the optimal path [4-6]. However, in practice, drivers consider not only the distance of the road but also the road characteristics, which are important factors to consider, such as the longitudinal slope, longitudinal length, the flat curve corner, pavement evenness, and pavement damage. These road characteristics have an important impact on the vehicle fuel economy and driving safety [7-12]. Especially in mountain cities, such as Qingdao and Chongqing in China and Boston in the United States, roads with the characteristics of large longitudinal slopes and small flat curve corners are common, which affect the choice of logistics distribution path, and should be considered in the modeling of distribution path planning and investigated in depth.

In addition, with the development of information and artificial intelligence, technologies related to intelligent driving have been continuously developed and applied, including three-dimensional high-precision maps applicable to vehicle navigation, which include road curvature, longitudinal slope, and other road characteristics information $[13,14]$. Moreover, with the development and improvement of the urban intelligent transportation system, the dynamic characteristics of road, such as pavement evenness, pavement damage, and pavement skid resistance, can be accessed by the road surface condition monitoring system $[15,16]$. These technologies and application system provide strong support for logistics distribution path planning considering road characteristics.

Under the background, the urban regional logistics distribution path planning considering the road characteristics is studied in the paper. The contributions of this study mainly involve the following three aspects:
(1) A mathematical model considering the road characteristics and distance is proposed. Different weight coefficients for the road characteristics and distance are analyzed, which illustrates that the model can produce a superior distribution path compared to considering the distance or road characteristics only.

(2) Based on the AHP method and the fuzzy comprehensive evaluation method, the road characteristics evaluation is presented to quantify the road characteristics.

(3) The adaptive genetic algorithm with a customized crossover operator is designed for the solution of model, which has better performance than genetic algorithm and is suitable for the logistics distribution path planning.

The rest of the paper is organized as follows. In Section 2, the research status of logistics distribution path planning is discussed. Section 3 presents a logistics distribution path mathematical model considering road characteristics and distance. In Section 4, the calculation method of the road characteristics evaluation value based on AHP and fuzzy comprehensive evaluation is presented. Section 5 designs the AGA for solving the model. Example for demonstration of the proposed model and algorithm is presented and discussed in Section 6. Finally, the conclusions and suggestions for future work are presented in Section 7.

\section{Literature Review}

Considering the available studies, we focus primarily on the factors and constraints considered in building a logistics distribution path mathematical model and solving methods. Logistics distribution path planning is a kind of VRP. VRP and its variants, vehicle routing problems with time windows (VRPTW), were studied earlier. Generally, the mathematical models for the VRP or VRPTW were formulated considering the vehicle travel distance, and the solutions to the models were the exact approaches, such as the linear programming, dynamic programming, and column generation. [17-21].

With the expansion of VRP application domain and the development of intelligent optimization algorithms, VRP and its variants have been further studied and several results have been achieved. Pisinger and Ropke [22] investigated five different variants of the VRP, which include VRPTW, the capacitated vehicle routing problem (CVRP), the multidepot VRP, the site-dependent VRP, and the open VRP. In the models, the constraints, such as time window, capacity, vehicle type, and number of distribution centers, were analyzed and the objective is to minimize the total traveled distance and the number of vehicles. A unified heuristic, adaptive large neighborhood search is presented to solve these models. Wang et al. [23] studied CVRP and built a model with the objective of minimizing the sum of the travel distances of all the vehicles. A hybrid particle swarm optimization algorithm is proposed to solve the CVRP model. Lau et al. [24] studied multidepot VRP and considered the traveling distance and time into the model. The objective is 
to minimize the total cost due to the distance traveled by all vehicles and the time required for all vehicles to serve customers. A fuzzy logic-guided genetic algorithm is proposed to solve the model. Wang et al. [25] studied a collaborative multiple-center VRP and established an integerprogramming model considering the transportation costs among distribution centers and vehicle routing costs in each distribution center. A multiphase hybrid approach with clustering, dynamic programming, and heuristic algorithm is presented to solve the model. Mohammed et al. [26] studied the CVRP considering the vehicle capacity and travel time. The objective is to minimize travel distance while ensuring customers' satisfaction. And genetic algorithm is used to determine the optimum vehicles route. Vidal et al. [27] introduced a mathematical model with the objective of minimizing the total travel distance for multiple periods VRPTW. A new hybrid genetic search with advanced diversity control is presented to efficiently address the model. Liao et al. [28] proposed a mathematical model for VRPTW considering the travel distance and vehicle waiting time for the customer, and the objective is to minimize total cost of transportation. A two-stage optimization algorithm with the help of ant-colony algorithm and plug-in heuristic algorithm is proposed to solve the model. Mohammed et al. [29] applied CVRP to a bus routing problem. A mathematical model was formulated considering the route distance. An improved GA is presented to solve the model. Chen et al. [4] established a biobjective mathematical model for mixedshift VRPTW, in which objectives are to minimize the total driver payment and the total travel distance. A hyperheuristic with two guidance indicators is proposed to resolve the model. Liu and Jiang [30] introduced the cumulative CVRP with time-window constraints, and the model is proposed with the objective of minimizing the sum of arrival times at all the customers and the fixed cost. Based on large neighborhood search algorithm and GA, an algorithm is proposed to solve the problem. Lei et al. [31] discussed the CVRP with stochastic demands and time windows and modeled the problem considering the vehicle travel cost. An adaptive large neighborhood search heuristic is developed for solution and assessed. Wang et al. [32] studied the multidepot green VRP and proposed a biobjective model with the objective of minimizing the total carbon emission and operating cost, in which the transportation cost is considered. A hybrid heuristic algorithm is designed to search the best vehicle routing solution.

In the abovementioned studies, the VRP and its variants are pick-up or delivery problem. There are many researchers who have studied vehicle routing problem considering pickup and delivery simultaneously, which is referred to as VRPSPD. Wang and Bian [33] studied the VRPSPD under different weights. A mixed integer linear programming model was formulated considering the distance transportation cost, inventory carrying costs, and shortage costs. The model was solved by using software Lingo. Lu et al. [6] proposed a model with the objective of minimizing the total routing costs defined as Euclidean distance for VRPSPD. A solution based on ant colony to solve the model and experimental evaluations shows the proposed solution had excellent performance. Zachariadis et al. [34] considered two-dimensional loading constraints and compared three transportation strategies: the VRPSPD, VRP with back hauls, and the bidirectional VRP. The model is proposed with the objective to minimize the total travel cost and solved by an optimization framework consisting of a local search method and a two-dimensional packing heuristic. Sitek and Wikarek [35] modeled VRPSPD considering the distances for couriers to travel and the penalty for delivering items to alternative points. A hybrid approach integrating mathematical programming and constraint logic programming was proposed to solve the model.

In addition, the vehicle routing problem with simultaneous pick-up and delivery and time windows (VRPSPDTW) and its variants has also received the attention of researchers, and a large number of results have emerged. Shi et al. [36] formulated the model of VRPSPDTW and the objective is to minimize the number of vehicles and the total traveling costs. An efficient Tabu search-based procedure was proposed, and the results showed it could produce better solutions. Lagos et al. [37] studied the VRPSPDTW, and a model with an objective of minimizing the total distance of paths was proposed. An improved swarm optimization algorithm was used to solve the model. Wang et al. [38] modeled the VRPSPDTW with the objective to minimize the traveling cost of vehicles. A parallel simulated annealing algorithm including a residual capacity and radial surcharge insertion-based heuristic is developed and applied to solve this problem. Wang and Chen [39] concerned a VRPSPDTW and proposed a model considering the dispatching cost of vehicle and total traveling cost. A coevolution GA with variants of the cheapest insertion method is developed to solve the model. Alaia et al. [5] studied VRPSPDTW with multidepots and multivehicles. The model was formulated considering the total travel distance, total tardiness time, and number of vehicles. A new algorithm based on the genetic algorithm was applied to the model and tested. Saâdia et al. [40] addressed the multitrip VRPSPDTW in a hospital complex. The objective is to minimize the traveling cost and the fixed cost of using vehicles, and GA algorithm is designed to solve the problem. In order to solve the problem of the optimization of pick-up and delivery cargoes' routes with time windows, Ma et al. [41] built a model with the objective of minimizing the transportation and time cost. The time cost is summation of all the time penalties, and the transportation cost is the function of distance. Tabu search algorithm was used to determine the order of pick-up and delivery points and the distribution route. Cherkesly et al. [42] studied the pick-up and delivery problem with time windows and last-in-first out loading considering the capacity constraints and time windows. The objective of the model is to minimize the number of vehicles and the total traveled distance. A population-based metaheuristic is proposed for solving the model.

The aforementioned works studied the VRP, VRPSPD, VRPSPDTW, and their variants. Their models were formulated considering various constrains and resolved the issues. However, most of the models focus on the objective function of the minimum vehicle travel cost considering the 


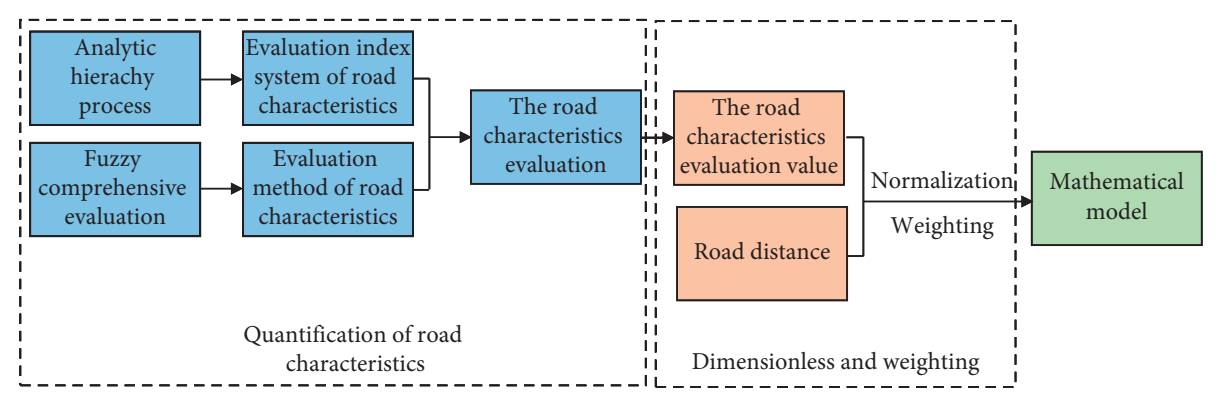

FIgURE 2: The block diagram for process of mathematical model establishment.

road distance. In practice, there are other factors that need to be considered in logistics distribution path planning. For example, a VRPTW mathematical model was established considering the road traffic volume which was expressed by the road-block function [43]. Simulation results indicate that the road traffic volume has significant influence on the optimal vehicle path of VRPTW and should be considered sufficiently. The CVRP taking the traffic density into account was studied, and GA was designed for the issue [44]. It is obtained that the delivery speed based on travel time is faster by almost two times compared to distance-based route search.

Meanwhile, the road characteristics have an important impact on the selection of logistics distribution path and are necessary to be investigated carefully. Consequently, in this paper, a mathematical model for logistics distribution path planning in urban area is proposed, which takes road characteristics into account. During the process, a road characteristics evaluation value is defined to quantify the road characteristics. To obtain the road characteristics evaluation value, an index system is established and the fuzzy comprehensive evaluation method is applied. Then, the mathematical model is obtained after normalization of the road characteristics evaluation value and the road distance.

\section{Mathematical Formulation}

3.1. Symbolic Definition. The sets, parameters, and decision variables used in this paper are defined as follows:

$J$ : set of all distribution stations, $J=\{1, \cdots, N\}$, where $N$ is the number of distribution stations

$S$ : set of node; nodes are distribution center and distribution stations in this paper, $S=\{0\} \cup J$, where 0 represents the distribution center

$H$ : distribution vehicle set, $H=\{1, \cdots, K\}$, where $K$ is the number of vehicles

$Q$ : the capacity of vehicle

$q_{i}$ : the demand of distribution station $i, i \in J$

$d_{i j}$ : the distance between node $i$ and node $j, i \in S, j \in S$, $i \neq j, d_{i j} \neq 0$

$e_{i j}$ : road characteristics evaluation value between node $i$ and node $j, i \in S, j \in S, i \neq j, e_{i j} \neq 0$

$\lambda_{1}$ and $\lambda_{2}$ : weight coefficients, $\lambda_{1}+\lambda_{2}=1$ $x_{i j k}$ : decision variable, $i \in S, j \in S, k \in H$; if the vehicle $k$ travels from node $i$ to node $j, x_{i j k}=1$; otherwise, $x_{i j k}=0$ $y_{i k}$ : decision variable, $i \in J, k \in H$; if the task of node $i$ is completed by vehicle $k, y_{i k}=1$; otherwise, $y_{i k}=0$

3.2. Model Establishment. In this paper, the road characteristics and distance are considered comprehensively into modeling the logistics distribution path planning in the urban area. The process of model establishment is shown in Figure 2, which mainly includes the quantification of road characteristics, the dimensionless and weighting of road characteristics evaluation value, and road distance. In the process, the key is to quantify the road characteristics. Through the evaluation of the factors and indexes related to road characteristics, the evaluation value of road characteristics can be obtained, which is defined as the road characteristics evaluation value. This enables a numerical representation of the road characteristics. In this paper, AHP and fuzzy comprehensive evaluation method are used to evaluate the road characteristics to obtain the evaluation value, which will be discussed in the next section of this paper. Here, it is assumed that the road characteristics evaluation value has been obtained, and a mathematical model is formulated first.

3.2.1. Dimensionless. Because the dimension of the road characteristics evaluation value and road distance are different, the normalization method is used to transform them to dimensionless. Normalization formulas for road distance and road characteristics evaluation value are as follows:

$$
d_{i j}^{\prime}=\frac{d_{i j}}{\max \left\{d_{i j}\right\}}, \quad(i=0,1,2, \cdots, N ; j=0,1,2, \cdots, N),
$$

where $\max \left\{d_{i j}\right\}$ represents the maximum distance between nodes.

$$
e_{i j}^{\prime}=\frac{e_{i j}}{\max \left\{e_{i j}\right\}}, \quad(i=0,1,2, \cdots, N ; j=0,1,2, \cdots, N),
$$

where $\max \left\{e_{i j}\right\}$ represents the maximum value of the road characteristics evaluation value between nodes. 
3.2.2. Mathematical Model. During the evaluation process of the road characteristics, the better the road characteristics, the higher the evaluation value; i.e., the path with a higher road characteristics evaluation value should be selected in the logistics distribution path. However, the shorter the road is, the more likely it is to be selected for the logistics distribution path. In order to obtain the minimum value as the objective function in the model, the normalized road characteristics evaluation value $e_{i j}^{\prime}$ is transformed to $1-e_{i j}^{\prime}$. Then through weighting the total of road characteristics evaluation value and the total road distance of all distribution paths, the mathematical model is obtained.

Assuming that $D_{1}$ and $D_{2}$, respectively, are total road distance and total road characteristics evaluation value of all distribution paths, they can be calculated as follows:

$$
\begin{aligned}
& D_{1}=\sum_{i \in S} \sum_{j \in S} \sum_{k \in H} d_{i j}^{\prime} x_{i j k}, \\
& D_{2}=\sum_{i \in S} \sum_{j \in S} \sum_{k \in H}\left(1-e_{i j}^{\prime}\right) x_{i j k} .
\end{aligned}
$$

Then, based on (3) and (4), the mathematical model is formulated as follows:

$$
\begin{gathered}
\min \quad \lambda_{1} D_{1}+\lambda_{2} D_{2}, \\
\text { Subject to: } \quad \sum_{i \in J} q_{i} y_{i k} \leq Q, \quad k \in H, \\
\sum_{i \in S} \sum_{k \in H} x_{i j k}=1, \quad j=1,2, \cdots, N, \\
\sum_{j \in S} \sum_{k \in H} x_{i j k}=1, \quad i=1,2, \cdots, N, \\
\sum_{i \in S} \sum_{k \in H} x_{0 i k}=\sum_{j \in S} \sum_{k \in H} x_{j 0 k}=K .
\end{gathered}
$$

Formula (5) is the objective function, which considers the road characteristics and distance comprehensively. Constraint (6) is vehicle load constraint. Constraints (7) and (8) require each distribution station must be serviced exactly once by only one vehicle. Constraint (9) enables the vehicle to return to the distribution center after completing the service of all distribution stations.

\section{Road Characteristics Evaluation}

The purpose of road characteristics evaluation is to get the road characteristics evaluation value. The road characteristics evaluation is composed of road characteristics evaluation index system and evaluation method of road characteristics. In the process, the AHP and fuzzy comprehensive evaluation method are used.

\subsection{Establishment of Road Characteristics Evaluation Index} System. There are many factors related to road characteristics, which can be divided into two categories: road plane factor and road profile factor. Once the road construction is completed, these factors will not change, which are called static factors.

As for the road plane factor, straight line is its basic element, which has the advantages of clear direction and shortest distance. However, with the increase in the straight line length, it will reduce the driving safety [45]. In addition, the circular curve radius, the flat curve corner, and the flat curve length, which belong to the plane factor, also affect the driver's operating strength and driving safety [9, 46, 47].

The road profile factor mainly includes road longitudinal slope, longitudinal length, vertical curve radius, and vertical curve length. They have an impact on the energy consumption of the vehicle; for example, when the longitudinal slope is large, the energy consumption of the vehicle will increase $[10,48]$ and also have an impact on the safe driving of the vehicle $[11,49]$.

In addition, the road conditions, such as the pavement evenness, pavement damage, and pavement skid resistance, also affect the fuel economy and driving comfort of the vehicle $[7,8,12]$. These factors will change with the use of the road and changing weather conditions. They are dynamic characteristics, which are considered to evaluate the road characteristics as described in this paper.

According to the above analysis, based on the AHP method, the road characteristics evaluation index system is obtained, and its structure is described in Figure 3. Among them, the road characteristics evaluation is the first layer, that is, the target layer; the second layer includes the road plane factor, road profile factor, and road condition, which is the criterion layer; the third layer is the subcriterion layer, that is, index layer.

4.2. Determination of Weight Vector. In the process of road characteristics evaluation, the determination of weight vector is a very important step, which includes establishment of judgment matrix, the consistency test of judgment matrix, and the calculation of weight vector.

4.2.1. Establishment of Judgment Matrix. Judgment matrix is an expression of relative importance for each factor in the same layer. The relative importance of each factor is obtained by comparison of each other. The nine-scale method is used to calculate the relative importance between factors, which is shown in Table 1 [50].

Suppose the number of factor in the criterion layer is $m$, the number of indexes for each criterion layer factor is $n_{i}$ $(i=1,2, \cdots, m)$. The forms of the judgment matrixes are expressed as follows.

Criterion layer judgment matrix $U$ :

\begin{tabular}{c|cccc}
$U$ & $U_{1}$ & $U_{2}$ & $\cdots$ & $U_{m}$ \\
\hline$U_{1}$ & $U_{11}$ & $U_{12}$ & $\cdots$ & $U_{1 m}$ \\
$U_{2}$ & $U_{21}$ & $U_{22}$ & $\cdots$ & $U_{2 m}$ \\
$\vdots$ & $\vdots$ & $\vdots$ & $\vdots$ & $\vdots$ \\
$U_{m}$ & $U_{m 1}$ & $U_{m 2}$ & $\cdots$ & $U_{m m}$
\end{tabular}




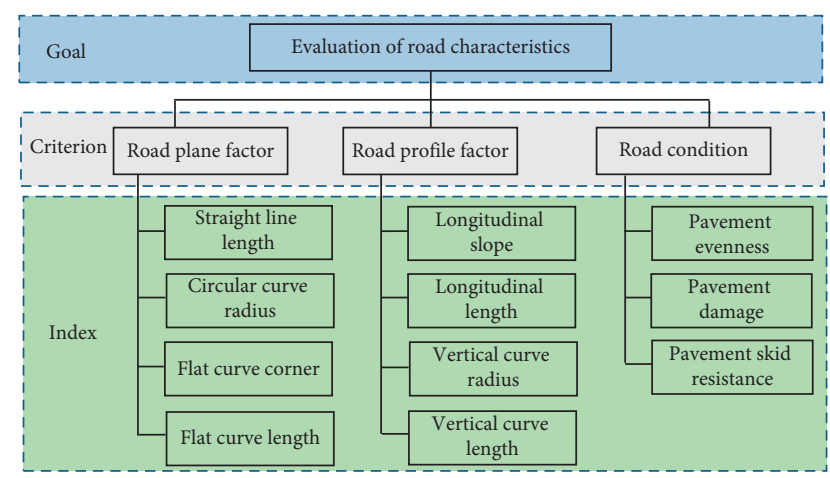

FIGURE 3: The structure of road characteristics evaluation index system.

TABle 1: Standard degree of the nine-scale method.

\begin{tabular}{lcc}
\hline Serial number & Order of importance & $U_{i j}$ value \\
\hline 1 & $i$ and $j$ are equally important & 1 \\
2 & $i$ is slightly more important than $j$ & 3 \\
3 & $i$ is significantly more important than $j$ & 5 \\
4 & $i$ is much more important than $j$ & 7 \\
5 & $i$ is more important than element $j$ & 9 \\
6 & $i$ is slightly less important than $j$ & $1 / 3$ \\
7 & $i$ is significantly less important than $j$ & $1 / 5$ \\
8 & $i$ is much less important than $j$ & $1 / 7$ \\
9 & $i$ is less important than $j$ & $1 / 9$ \\
\hline
\end{tabular}

Index layer judgment matrix $U_{i}$ for the $i$ factor in the criterion layer:

\begin{tabular}{c|cccc}
$U_{i}$ & $U_{i 1}$ & $U_{i 2}$ & $\cdots$ & $U_{i n_{i}}$ \\
\hline$U_{i 1}$ & $U_{i 1, i 1}$ & $U_{i 1, i 2}$ & $\cdots$ & $U_{i 1, n_{i}}$ \\
$U_{i 2}$ & $U_{i 2, i 1}$ & $U_{i 2, i 2}$ & $\cdots$ & $U_{i 2, i n_{i}}$ \\
$\vdots$ & $\vdots$ & $\vdots$ & $\vdots$ & $\vdots$ \\
$U_{i n_{i}}$ & $U_{i n_{i}, i 1}$ & $U_{i n_{i}, i 2}$ & $\cdots$ & $U_{i n_{i}, i n_{i}}$
\end{tabular}

4.2.2. Consistency Test of Judgment Matrix. The consistency index CI is used for the consistency test of the judgment matrix and is calculated as follows [51]:

$$
\mathrm{CI}=\frac{\lambda_{\max }-n}{n-1},
$$

where $\lambda_{\max }$ is the maximum eigenvalue of the judgment matrix and $n$ is the size of the judgment matrix.

The random consistency ratio (CR) is adopted to qualify the consistency, and its calculation formula is as follows:

$$
\mathrm{CR}=\frac{\mathrm{CI}}{\mathrm{RI}}
$$

where RI is the random consistency index; its value has been defined in [52], in which it ranges from 0 to 1.49 corresponding to $n$ from 2 to 10 based on matrix size.

After calculation, if $\mathrm{CR}<0.1$, the consistency of judgment matrix is qualified; otherwise, the judgment matrix needed to be adjusted.
4.2.3. Calculation of the Weight Vector. According to the judgment matrices (10) and (11), the arithmetic averaging method is used to calculate the weight vectors of the criterion layer and the index layer.

The calculation formula for weight vector of the criterion layer $\theta_{i}$ is as follows:

$$
\theta_{i}=\frac{1}{m} \sum_{j=1}^{m} \frac{U_{i j}}{\sum_{k=1}^{m} U_{k j}}, \quad(i=1,2, \cdots, m ; j=1,2, \cdots, m) .
$$

The calculation formula for weight vector of the index layer $\omega_{i k}$ is as follows:

$$
\omega_{i k}=\frac{1}{n_{i}} \sum_{j=1}^{n_{i}} \frac{U_{i k, i j}}{\sum_{k=1}^{n_{i}} U_{i k, i j}}, \quad\left(i=1,2, \cdots, n_{i} ; j=1,2, \cdots, n_{i}\right) .
$$

According to (14) and (15), the weight vector of the criterion layer for road characteristics evaluation index system is obtained: $\theta=\left(\theta_{1}, \theta_{2}, \theta_{3}\right)$, and the weight vectors of the index layer are obtained: $\omega_{1}=\left(\omega_{11}, \omega_{12}, \omega_{13}, \omega_{14}\right)$, $\omega_{2}=\left(\omega_{21}, \omega_{22}, \omega_{23}, \omega_{24}\right)$, and $\omega_{3}=\left(\omega_{31}, \omega_{32}, \omega_{33}\right)$.

4.3. Evaluation Method of Road Characteristics. Because the fuzzy comprehensive evaluation method has the characteristics of clear results and strong systematization, in this paper, it is used to determine the road characteristics evaluation value.

4.3.1. Establishment of the Road Characteristics Evaluation Set. The evaluation set is comprised of various possible evaluation levels about road characteristics. Based on [53], the road characteristics evaluation set $V$ is established, which includes five levels: $V_{1}, V_{2}, V_{3}, V_{4}$, and $V_{5}$, and their corresponding score ranges are given, as shown in Table 2.

4.3.2. Determination of Membership Degree Function. According to Table 2, the trapezoid and triangle fuzzy functions are used as the membership degree functions, as shown in Figure 4 . In the figure, functions $r_{1}, r_{2}, r_{3}, r_{4}$, and $r_{5}$ are the membership functions of the road characteristics, which correspond to "bad," "general," "medium," "good," and "excellent" level, respectively.

4.3.3. Establishment of Fuzzy Evaluation Matrix. According to Figure 4, the membership degree of each index is determined. Then, the membership matrix $R_{i}$ of the criterion $i$ is as follows:

$$
R_{i}=\left[\begin{array}{cccc}
r_{i 11} & r_{i 12} & \cdots & r_{i 15} \\
r_{i 21} & r_{i 22} & \cdots & r_{i 25} \\
\vdots & \vdots & \vdots & \vdots \\
r_{i n_{i} 1} & r_{i n_{i} 2} & \cdots & r_{i n_{i} 5}
\end{array}\right],
$$


TABLE 2: Road characteristics evaluation set.

\begin{tabular}{lcccc}
\hline$V_{1}$ & $V_{2}$ & $V_{3}$ & $V_{4}$ & $V_{5}$ \\
\hline Bad & General & Medium & Good & Excellence \\
$(0-20)$ & $(20-40)$ & $(40-60)$ & $(60-80)$ & $(80-100)$ \\
\hline
\end{tabular}

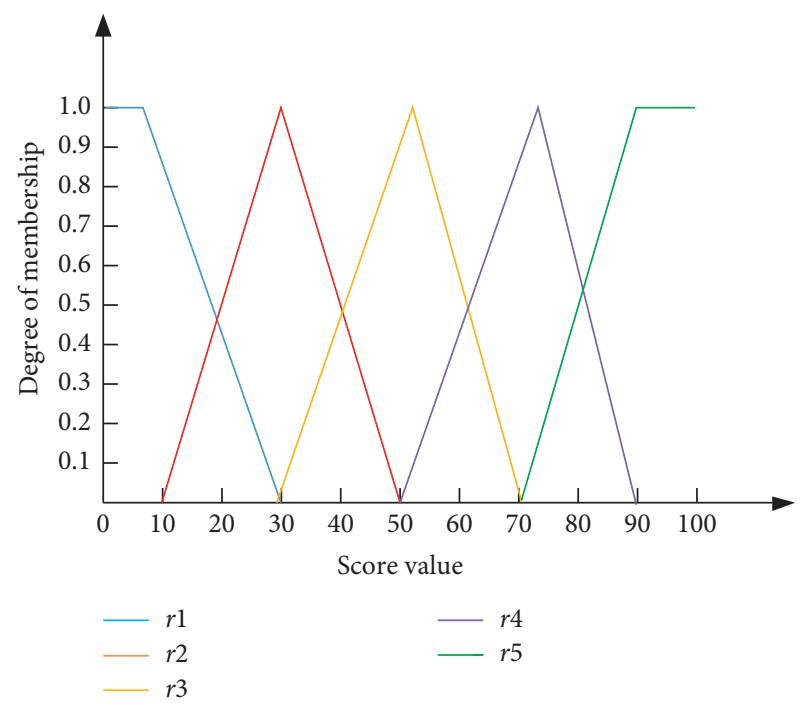

FIgUre 4: Membership degree functions of road characteristics.

where $r_{i j k}\left(i=1,2,3 ; j=1, \cdots, n_{i} ; k=1,2, \cdots, 5\right)$ is the membership degree of the $k$ level in the evaluation set for index $j$ of criterion $i$.

The comprehensive evaluation vector of the criterion layer can be obtained by multiplying each column of elements based on their weight vectors, that is,

$$
B_{i}=\omega_{i} \cdot R_{i}=\left[b_{i 1}, b_{i 2}, \cdots, b_{i 5}\right] \text {, }
$$

and the comprehensive evaluation matrix $B$ for the criterion layer is obtained: $B=\left[B_{1}, B_{2}, B_{3}\right]^{\mathrm{T}}$; then, based on the matrix $B$, the comprehensive evaluation vector $C$ for the goal layer is calculated by fuzzy transformation, that is,

$$
\begin{aligned}
C & =\theta \cdot B \\
& =\left[\begin{array}{lll}
\theta_{1} & \theta_{2} & \theta_{3}
\end{array}\right] \cdot\left[\begin{array}{c}
B_{1} \\
B_{2} \\
B_{3}
\end{array}\right] \\
& =\left[\begin{array}{llllll}
\theta_{1} & \theta_{2} & \theta_{3}
\end{array}\right] \cdot\left[\begin{array}{lllll}
b_{11} & b_{12} & b_{13} & b_{14} & b_{15} \\
b_{21} & b_{22} & b_{23} & b_{24} & b_{25} \\
b_{31} & b_{32} & b_{33} & b_{34} & b_{35}
\end{array}\right] \\
& =\left[\begin{array}{lllll}
C_{1} & C_{2} & C_{3} & C_{4} & C_{5}
\end{array}\right] .
\end{aligned}
$$

4.3.4. Calculation of Road Characteristics Evaluation Value. According to the principle of maximum comprehensive membership degree, the road characteristics levels corresponding to the maximum membership degree and the submaximum membership degree can be obtained. Then, the road characteristics evaluation value $e$ is calculated, and the specific process is as follows: (i) According to the comprehensive evaluation vector $C$ of the goal layer, the maximum membership degree and submaximum membership degree are obtained, supposed as $C_{k}$ and $C_{m}$, respectively $(k$, $m=1,2, \cdots, 5)$.

(ii) Road characteristics levels $V_{k}$ and $V_{m}$ are obtained according to $C_{k}$ and $C_{m}$.

(iii) If $k=1$ or $k=5$, the score of the road characteristics can be obtained from $C_{k}$ and $V_{k}$ based on Figure 4; that is, the road characteristics evaluation value $e$ is obtained.

(iv) If $k=2$ or $k=3$ or $k=4$, two score values of road characteristics are obtained from $C_{k}$ and $V_{k}$ based on Figure 4, supposed as $e_{1}$ and $e_{2}$, and set $e_{1}<e_{2}$; if $k<m, e=e_{2}$; otherwise, $e=e_{1}$.

According to the above process, the all road characteristics evaluation value $e_{i j}(i=0,1,2, \cdots, N ; j=0,1,2, \cdots$, N) can be obtained.

\section{Algorithms for the Model}

5.1. Adaptive Genetic Algorithm. For several decades, the algorithms for solving vehicle routing problem have been studied extensively around the world. Because of its good concurrency performance, GA has been widely used to resolve VRP and its variants [54, 55]. However, in the traditional GA, the performance of the algorithm is affected due to the fixed cross probability and mutation probability. Therefore, many scholars have applied the AGA to the vehicle routing problem [56-59].

In GA, if cross probability is too large, it will destroy the genetic model; if cross probability is too small, it will slow the search or even stop the search in serious cases. Besides, if mutation probability is too small, the new individual structure will not be easy to produce; if mutation probability is too large, GA will lose its characteristics and become a simple random search algorithm. However, in the AGA, the adaptive cross probability and mutation probability can be adjusted according to the fitness value of the individual. The adaptive cross probability $p_{c}$ and mutation probability $p_{m}$ are adjusted according to the following formula:

$$
\begin{aligned}
& p_{c}= \begin{cases}\frac{k_{1}\left(f_{\max }-f^{\prime}\right)}{f_{\text {max }}-f_{\text {avg }}}, & f^{\prime} \geq f_{\text {avg }}, \\
k_{3}, & f^{\prime}<f_{\text {avg }},\end{cases} \\
& p_{m}= \begin{cases}\frac{k_{2}\left(f_{\max }-f\right)}{f_{\text {max }}-f_{\text {avg }}}, & f \geq f_{\text {avg }}, \\
k_{4}, & f<f_{\text {avg }},\end{cases}
\end{aligned}
$$

where $f_{\max }$ is the maximum fitness in each generation of population, $f_{\text {avg }}$ is the average value of population fitness for each generation, $f^{\prime}$ is the larger fitness value of the two individuals, and $f$ is the fitness value of the individual to be mutated; $k_{1}, k_{2}, k_{3}$, and $k_{4} \in(0,1)$. 
The flowchart of the AGA is shown in Figure 5.

\subsection{The Design of AGA}

5.2.1. Coding and Chromosome. Natural number coding method is used, which simplifies the process of decoding after obtaining the optimal chromosome and reduces the volume of computation. The distribution stations are represented by natural numbers $1,2,3, \cdots, N$ in the chromosome, and the distribution center is represented by 0 . The vehicle starts from the distribution center and returns after completing the service at distribution stations. The form of a distribution path is

$$
\text { " } 0 n_{1} n_{2} \cdots n_{i} 0 \text { ", }
$$

where $n_{1}, n_{2}, \cdots, n_{i}$ is the natural number in $\{1,2,3, \cdots, N\}$.

If the number of distribution vehicles is $K, K$ paths should be planned; that is, $K$ paths would be the same form: " $0 n_{1} n_{2} \cdots n_{i} 0$." From this, it is obtained that the chromosome length in the natural coding mode is $K+1+N$, and the form of chromosome is

$$
\text { " } 0 n_{1} n_{2} \cdots n_{i} 0 n_{i+1} n_{i+2} \cdots 0 \cdots 0 n_{j} \cdots n_{N} 0 ",
$$

where the number of distribution center 0 is $K+1$, and $n_{1}$, $n_{2}, \cdots, n_{i}, \cdots, n_{j}, \cdots, n_{N}$ are combinations of distribution stations $1,2,3, \cdots, N$.

5.2.2. Fitness Function. In AGA, the fitness function is used to measure the adaptability of each individual in the population. The objective function (5) is to find the minimum value, and its reciprocal is used as the fitness function of chromosome.

5.2.3. Design of Genetic Operator. Genetic operators include selection, crossover, and mutation, which in turn acts on the population and results in a new generation of population.

(1) Selection Operator. The fitness proportional model, also known as the gambling or Monte Carlo selection, is used as selection operator. The method is a playback random sampling method, and selection probability of each individual is proportional to its fitness value.

(2) Crossover Operator. The function of crossover operator is to produce diverse chromosomes. In the chromosome, the form of which is " $0 n_{1} n_{2} \cdots n_{i}, 0 n_{i+1} n_{i+2} \cdots 0 \cdots 0, n_{j} \cdots n N$ 0 ," the genes represent distribution stations and distribution center, and their adjacency relation and sequence represent distribution path. In order to maintain this adjacency between genes and produce various chromosomes, a crossover operator is customized. In crossover operation, the position of crossover point is selected according to the distribution path in the chromosome; that is, one distribution path is randomly selected in the parent chromosome and kept in offspring, and other gene positions of offspring are filled by different genes of another parent chromosome, so as to obtain the offspring chromosome. The process of crossover

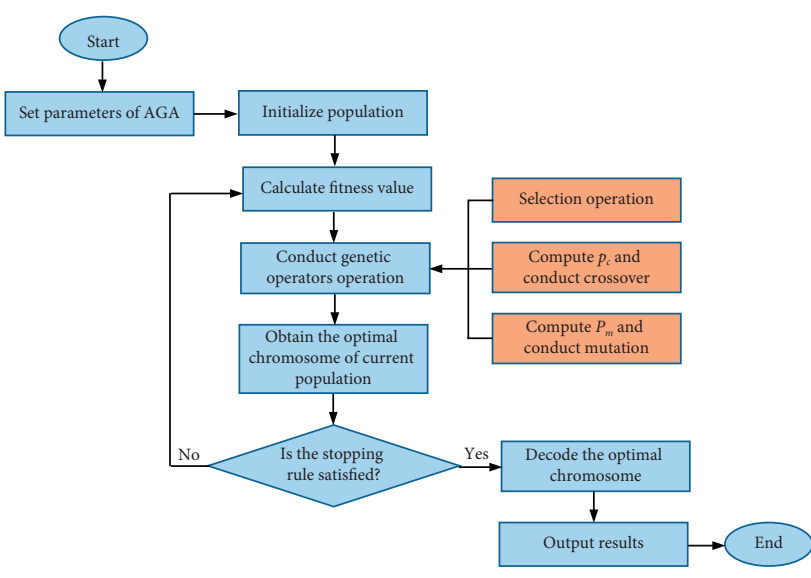

FIgURe 5: Flowchart of AGA.

operation with four distribution paths and eleven distribution stations in chromosome is shown in Figure 6. It should be noted that one offspring chromosome is generated, as shown in Figure 6, and another one is generated in the same way. In addition, the crossover probability is calculated by (19) during the crossover operation.

(3) Mutation Operator. The mutation operator adopts the method of reverse mutation. That is, in the process of mutation, an inversion region composed of several genes is randomly selected, and then the value of the genes in the region are reversed and inserted into the original position again. The mutation probability is calculated by (20).

5.2.4. Stopping Rule of Algorithm. When the algorithm reaches the maximum number of iteration, the algorithm stops and outputs the optimal solution; otherwise, the algorithm enters the next iteration cycle.

\section{Simulation Experiment}

6.1. Example Analysis. Qingdao is a mountain city, in which the roads with the characteristics of large longitudinal slopes and small flat curve corners are common, and has good spatial accessibility [60-62]. A real logistics distribution path planning example in an urban area of Qingdao is selected to verify the logistics distribution path mathematical model proposed in the paper. In this example, there is one distribution center and 11 distribution stations. According to the location relationship between the distribution center and each distribution station, the topological map is obtained, as shown in Figure 7 . In the figure, $C_{0}$ represents the distribution center, $S_{1}, S_{2}, \cdots, S_{11}$ represent the distribution stations, and the numbers on the line represent the actual distance (unit: $\mathrm{km}$ ) between distribution stations and center. The demands (unit: ton) of distribution stations $S_{1}, S_{2}, \cdots, S_{11}$ are $1.54,1.65,1.3,1.23,1.54,1.55,1.42,0.91,1.4,1.23$, and 1.86 , respectively. The capacity of vehicle is 5 tons.

According to formula (5), the logistics distribution path planning mathematical model of the example is obtained. In the model, the distances between the distribution center and 


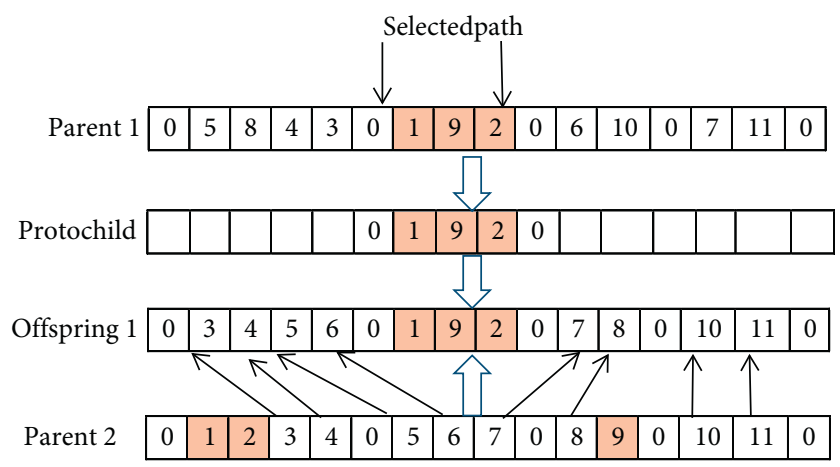

FIgURE 6: The process of crossover operation.

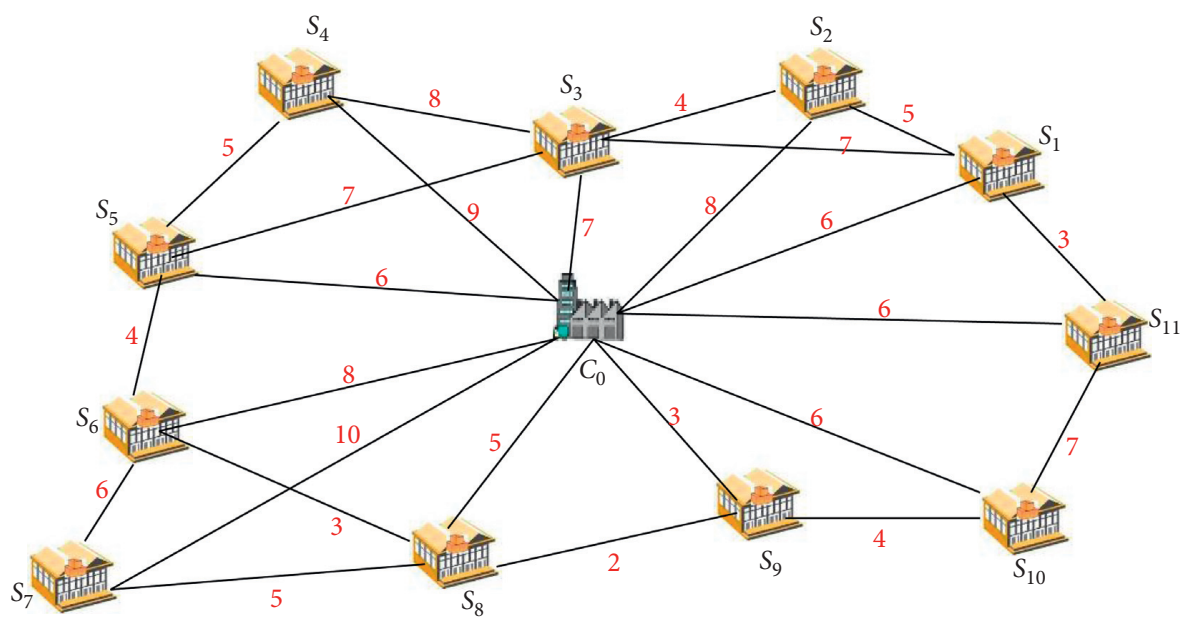

Figure 7: Distribution center and distribution station topology map.

TABLE 3: Evaluation index values.

\begin{tabular}{lccc}
\hline Factor & Index name & Unit & Index value \\
\hline \multirow{4}{*}{ Plane factor } & Straight line length & $m$ & 1800 \\
& Circular curve radius & $m$ & 400 \\
& Flat curve corner & $\circ$ & 15 \\
& Flat curve length & $m$ & 260 \\
\hline \multirow{5}{*}{ Profile factor } & Longitudinal slope & & 1.3 \\
& Longitudinal length & $m$ & 200 \\
& Vertical curve radius & $m$ & 100 \\
& Vertical curve length & $m$ & 50 \\
\hline \multirow{3}{*}{ Road condition } & Pavement evenness & & Better \\
& Pavement damage & & Better \\
& Pavement skid resistance & & Better \\
\hline
\end{tabular}

stations are known, but the road characteristics value is needed to be calculated.

Taking the road between $C_{0}$ and $S_{1}$ (labeled as $C_{0}-S_{1}$ ), as shown in Figure 7 as an example, a road characteristics evaluation value $e_{01}$ is calculated based on the evaluation index system and the calculation method presented in Section 4 of this paper. The evaluation indexes and their values in the road evaluation index system are shown in Table 3.

The importance of each index in Table 3 was scored by experts in the field, and then according to (10) and (11), the criterion layer and index layer judgment matrices are obtained:

$$
\begin{aligned}
U & =\left[\begin{array}{ccc}
1 & 7 & 3 \\
1 / 7 & 1 & 1 / 7 \\
1 / 3 & 7 & 1
\end{array}\right], \\
U_{1} & =\left[\begin{array}{cccc}
1 & 1 / 5 & 1 / 7 & 1 / 5 \\
7 & 1 & 5 & 7 \\
7 & 1 / 5 & 1 & 3 \\
5 & 1 / 7 & 1 / 3 & 1
\end{array}\right], \\
U_{2} & =\left[\begin{array}{cccc}
1 & 1 / 5 & 1 & 5 \\
5 & 1 & 5 & 7 \\
1 & 1 / 5 & 1 & 5 \\
1 / 5 & 1 / 7 & 1 / 5 & 1
\end{array}\right], \\
U_{3} & =\left[\begin{array}{ccc}
1 & 1 / 5 & 1 / 3 \\
5 & 1 & 1 \\
3 & 1 & 1
\end{array}\right] .
\end{aligned}
$$


According to (12) and (13), the judgment matrices (21) satisfy the term: $C R<0.1$, which meet the consistency test.

Based on (14) and (15), the weight vectors for the criterion layer and the index layer are determined, which are as follows:

$$
\begin{aligned}
\theta & =[0.623,0.066,0.311], \\
\omega_{1} & =[0.047,0.605,0.227,0.130], \\
\omega_{2} & =[0.164,0.624,0.164,0.048], \\
\omega_{3} & =[0.115,0.480,0.405] .
\end{aligned}
$$

According to the membership function shown in Figure 4 and (16), the membership matrices of index layer are obtained as follows:

$$
\begin{aligned}
& R_{1}=\left[\begin{array}{ccccc}
0 & 0.25 & 0.75 & 0 & 0 \\
0 & 0 & 0.75 & 0.25 & 0 \\
0 & 0 & 0.8 & 0.2 & 0 \\
0 & 0.7 & 0.3 & 0 & 0
\end{array}\right], \\
& R_{2}=\left[\begin{array}{llccc}
0 & 0 & 0.7 & 0.3 & 0 \\
0 & 0 & 0.8 & 0.2 & 0 \\
0 & 0 & 0.85 & 0.15 & 0 \\
0 & 0.2 & 0.8 & 0 & 0
\end{array}\right], \\
& R_{3}=\left[\begin{array}{lllll}
0 & 0.15 & 0.85 & 0 & 0 \\
0 & 0 & 0.25 & 0.75 & 0 \\
0 & 0 & 0.15 & 0.75 & 0
\end{array}\right] .
\end{aligned}
$$

According to (17), the fuzzy comprehensive evaluation matrix for the criterion layer is obtained as follows:

$$
B=\left[\begin{array}{lllll}
0 & 0.038 & 0.765 & 0.197 & 0 \\
0 & 0.010 & 0.790 & 0.199 & 0 \\
0 & 0.017 & 0.279 & 0.704 & 0
\end{array}\right] .
$$

According to (18), the comprehensive evaluation vector $C$ for the goal layer is obtained as follows:

$$
C=[0,0.029,0.622,0.349,0],
$$

Based on the calculation method of the road characteristics evaluation value, the maximum membership degree and the submaximum membership degree in $C$ are $C_{3}$ and $C_{4}$, respectively, and the corresponding road characteristics levels are $V_{3}$ and $V_{4}$. As described in Figure 4, the two score values of road characteristics corresponding to $C_{3}$ are $e_{1}=45$ and $e_{2}=60$, since $k=3, m=4$, and $k<m$; then, $e_{2}$ is selected as road characteristics evaluation value for $C_{0}-S_{1}$, namely, $e_{01}=60$.

Similarly, the other road characteristics evaluation values between the distribution center and stations in Figure 7 can be calculated, as shown in Table 4 .

6.2. Simulation Results and Discussion. Chromosome length is an important parameter of AGA. In this paper, the chromosome is encoded by natural number, and its length is related to the number of vehicles. According to the demands of distribution stations and the capacity of the vehicle, the required number of vehicles $K$ is calculated as follows:

$$
K=\left[\frac{\sum_{i=1}^{N} q_{i}}{Q}\right] .
$$

Based on the number of vehicle $K$, the length and form of chromosome are obtained. The other parameters of the AGA are set as follows: population size is 100 , number of iterations is 1000 , and crossover and mutation probability are calculated by (19) and (20), respectively, where $k_{2}$ and $k_{4}$ are 0.5 and $k_{1}$ and $k_{3}$ are 1.0 .

According to objective function (5), the road characteristics and distance are considered equally at first. In the case, the weight coefficients $\lambda_{1}$ and $\lambda_{2}$ are set as 0.5 . The simulation is carried out, and the results are obtained, as shown in Table 5, in which the objective value is the total of the road distance and road characteristics evaluation value of all distribution paths.

From Table 5, four distribution paths are obtained, and each path enables the vehicle to proceed from the distribution center and return to the distribution center after completing a certain task. Each load of distribution vehicle does not exceed its capacity, which satisfies the load constraint of the distribution vehicle. The total road distance is 4.76, and the total road characteristics value is 2.25.

Figure 8 shows the convergence curves of GA and AGA. It shows that the AGA is superior to GA in the convergence speed and the optimal solution obtained and indicates the AGA is an effective method for solving the logistics distribution path optimization problem.

In order to investigate the different influences of road characteristics and distance on the optimal distribution path, the simulations have been conducted under two special cases: first, considering road distance only; second, considering road characteristics only. In the two cases, the weight coefficients $\lambda_{1}$ and $\lambda_{2}$ are $\lambda_{1}=0$ and $\lambda_{2}=1$ and $\lambda_{1}=1$ and $\lambda_{2}=0$, respectively. After the simulations, the results are obtained and shown in Tables 6 and 7.

As shown in Tables 6 and 7, all the distribution paths obtained are feasible and four vehicles start from distribution center and return to the distribution center after completing all the requirements of distribution stations with their loads not exceeding the capacity. However, the total of the road characteristics evaluation value in Table 6 is smaller than that in Table 7. On the contrary, the total road distance in Table 7 is smaller than that in Table 6. This indicates the optimal path with better road characteristics is obtained in the case of considering road characteristics only, and in the case of considering road distance only, the optimal path with a smaller road distance is obtained. About the total of the objective value, it is smaller in Table 7 than Table 6. This result is due to the improvement of road distance in Table 7 more than the improvement of road characteristics in $\mathrm{Ta}$ ble 6 in the example.

Furthermore, the results in Table 5 are compared with that in Tables 6 and 7. In terms of the total objective value, the smallest is in Table 5, followed by Table 7, and the largest 
TABLE 4: Road characteristics evaluation values.

\begin{tabular}{lccc}
\hline Road & Road characteristics evaluation values & Road & Road characteristics evaluation values \\
\hline$C_{0}-S_{2}$ & 80 & $S_{2}-S_{3}$ & 100 \\
$C_{0}-S_{3}$ & 100 & $S_{4}-S_{5}$ & 100 \\
$C_{0}-S_{4}$ & 80 & $S_{1}-S_{3}$ & 90 \\
$C_{0}-S_{5}$ & 90 & $S_{5}-S_{6}$ & 100 \\
$C_{0}-S_{6}$ & 80 & $S_{6}-S_{7}$ & 90 \\
$C_{0}-S_{7}$ & 80 & $S_{3}-S_{5}$ & 90 \\
$S_{7}-S_{8}$ & 90 & $S_{8}-S_{9}$ & 70 \\
$S_{6}-S_{8}$ & 90 & $S_{10}-S_{11}$ & 80 \\
$C_{0}-S_{8}$ & 70 & $S_{1}-S_{2}$ & 80 \\
$C_{0}-S_{9}$ & 90 & $S_{11}-S_{1}$ & 100 \\
$C_{0}-S_{10}$ & 60 & $S_{9}-S_{10}$ & 100 \\
$C_{0}-S_{11}$ & 90 & $S_{3}-S_{5}$ & 70 \\
\hline
\end{tabular}

TABLE 5: Distribution paths and related results in the case of $\lambda_{1}=\lambda_{2}=0.5$.

\begin{tabular}{lcccc}
\hline Distribution path & Load (ton) & Distance & Road characteristics evaluation value & Objective value of each path \\
\hline $0 \rightarrow 7 \rightarrow 8 \rightarrow 10 \rightarrow 9 \rightarrow 0$ & 4.96 & 1.44 & 0.65 & 2.09 \\
$0 \rightarrow 3 \rightarrow 2 \rightarrow 0$ & 2.95 & 1.05 & 0.2 & 1.25 \\
$0 \rightarrow 6 \rightarrow 5 \rightarrow 4 \rightarrow 0$ & 4.32 & 1.44 & 0.7 & 2.14 \\
$0 \rightarrow 11 \rightarrow 1 \rightarrow 0$ & 3.4 & 0.83 & 0.7 & 1.53 \\
\hline Total & $\mathbf{1 5 . 6 3}$ & $\mathbf{4 . 7 6}$ & $\mathbf{2 . 2 5}$ & $\mathbf{7 . 0 1}$ \\
\hline
\end{tabular}

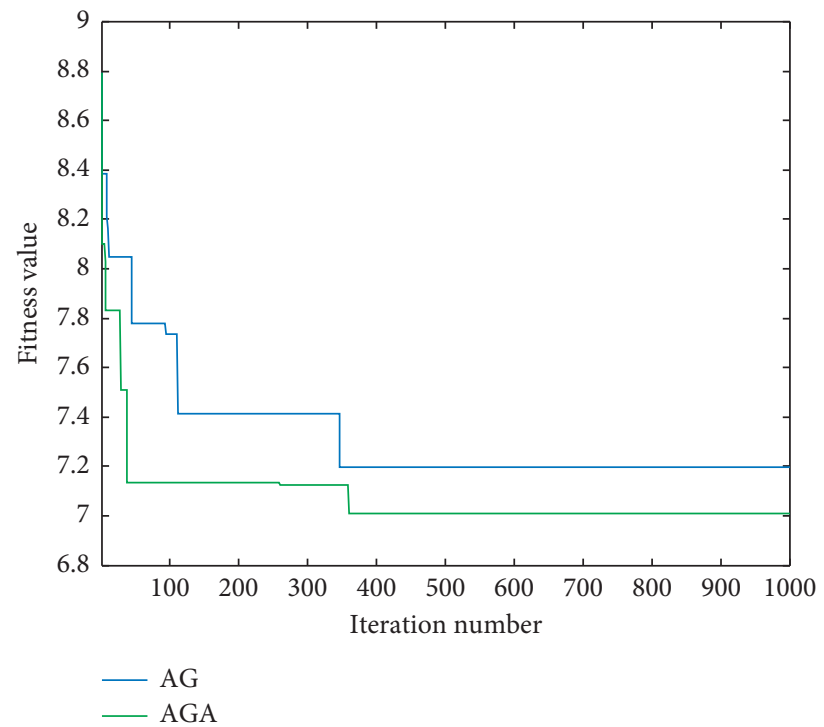

FIgURE 8: The convergence curves of GA and AGA.

TABLE 6: Distribution paths and related results in the case of $\lambda_{1}=0$ and $\lambda_{2}=1$.

\begin{tabular}{lcccc}
\hline Distribution path & Load (ton) & Distance & Road characteristics evaluation value & Objective value of each path \\
\hline $0 \rightarrow 11 \rightarrow 0$ & 1.86 & 0.66 & 0.4 & 1.06 \\
$0 \rightarrow 7 \rightarrow 8 \rightarrow 10 \rightarrow 9 \rightarrow 0$ & 4.96 & 1.44 & 0.65 & 2.09 \\
$0 \rightarrow 2 \rightarrow 5 \rightarrow 6 \rightarrow 0$ & 4.74 & 1.71 & 0.75 & 2.46 \\
$0 \rightarrow 3 \rightarrow 1 \rightarrow 4 \rightarrow 0$ & 4.07 & 2.11 & 0.4 & 2.51 \\
\hline Total & $\mathbf{1 5 . 6 3}$ & $\mathbf{5 . 9 2}$ & $\mathbf{2 . 2}$ & $\mathbf{8 . 1 2}$ \\
\hline
\end{tabular}


TABLe 7: Distribution paths and related results in the case of $\lambda_{1}=1$ and $\lambda_{2}=0$.

\begin{tabular}{lcccc}
\hline Distribution path & Load (ton) & Distance & Road characteristics evaluation value & Objective value of each path \\
\hline $0 \rightarrow 10 \rightarrow 8 \rightarrow 9 \rightarrow 0$ & 3.54 & 0.83 & 1 & 1.83 \\
$0 \rightarrow 4 \rightarrow 3 \rightarrow 2 \rightarrow 0$ & 4.18 & 1.6 & 0.5 & 2.1 \\
$0 \rightarrow 11 \rightarrow 1 \rightarrow 0$ & 3.40 & 0.83 & 0.7 & 1.53 \\
$0 \rightarrow 5 \rightarrow 6 \rightarrow 7 \rightarrow 0$ & 4.51 & 1.44 & 0.9 & 2.34 \\
\hline Total & $\mathbf{1 5 . 6 3}$ & $\mathbf{4 . 7}$ & $\mathbf{3 . 1}$ & $\mathbf{7 . 8}$ \\
\hline
\end{tabular}

TABLE 8: The results under different values of $\lambda_{1}$ and $\lambda_{2}$.

\begin{tabular}{lccc}
\hline Weight coefficients and their values & Total distance & Total road characteristics evaluation value & Total objective value of all paths \\
\hline$\lambda_{1}=0.2, \lambda_{2}=0.8$ & 5.76 & 2.3 & 8.06 \\
$\lambda_{1}=0.4, \lambda_{2}=0.6$ & 5.16 & 2.4 & 7.56 \\
$\lambda_{1}=0.6, \lambda_{2}=0.4$ & 5.00 & 2.6 & 7.6 \\
$\lambda_{1}=0.8, \lambda_{2}=0.2$ & 4.81 & 2.9 & 7.71 \\
\hline
\end{tabular}

in Table 6. It indicates that the optimal distribution path obtained by model considering the road distance and road characteristics is better than that obtained by model considering road distance only or road characteristics only.

In summary, the total distance in the case considering the road distance and road characteristics is increased by about $1.2 \%$, while the total objective value is reduced by $10.1 \%$ compared with the case of considering the road distance only. And the total road characteristics evaluation value in the case considering the distance and road characteristics is increased by $2.3 \%$, while the total objective value decreased by $13.7 \%$ compared with the case of considering road characteristics only. This shows that the distribution path with better cost performance is obtained by considering the distance and road characteristics.

In addition, the total distance is smallest in Table 7 , followed by Table 5 , and the largest in Table 6 , and the total road characteristics evaluation value is smallest in Table 6, followed by Table 5, and the largest in Table 7 . It shows that if the model only considers the road distance, the distance of shortest paths can be obtained, but the road characteristics are poor. At the same time, if the model only considers the road characteristics, the paths with the best road characteristics can be obtained, but it brings the increase in road distance.

In order to further investigate the influences of road characteristics and distance on the optimal distribution path, other more cases, in which $\lambda_{1}$ is set as $0.2,0.4,0.6$, and 0.8 , respectively (corresponding value of $\lambda_{2}$ is $0.8,0.6,0.4$, and 0.2 ), are simulated, and the results are shown in Table 8 . Based on Table 8 and other three cases results from Table 5 to Table 7, all the results are shown in Figure 9.

From Figure 9 and Table 8, it can be found that, with the gradual decrease in $\lambda_{2}$, the trend of total distance decreases and the total road characteristics evaluation value increases. With this changing trend of $\lambda_{2}$, when $\lambda_{2}=0$, that is, the model only considers the road distance, and the road distance of the optimal path is the smallest, which can be verified from Table 7 . On the contrary, with the gradual decrease in $\lambda_{1}$, the trend of total road characteristics evaluation value decreases and the total road distance increases,

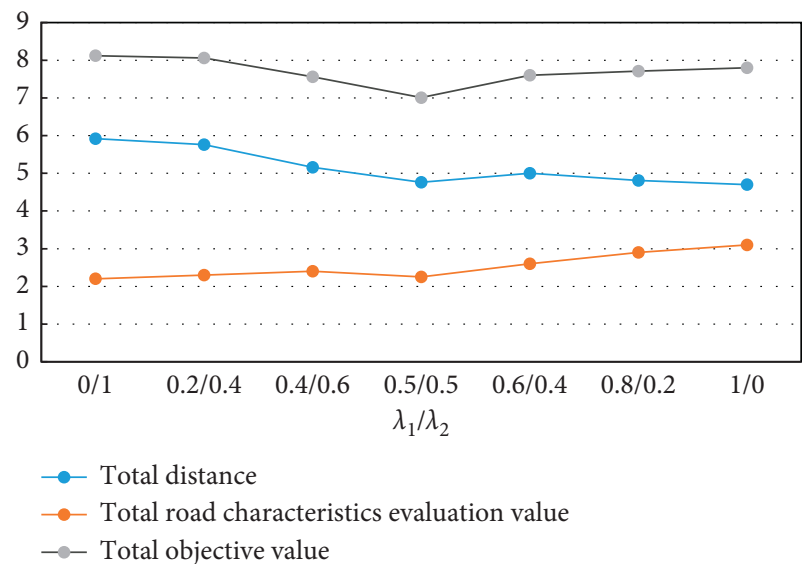

Figure 9: The results under all cases.

and when $\lambda_{1}=0$, that is, the model only considers the road characteristics, the total road characteristics evaluation value of the optimal path is the smallest, which can be verified from Table 6.

Furthermore, from the curve of the total objective value shown in Figure 9, it shows that the total objective value in the cases with $\lambda_{1} \neq 0$ and $\lambda_{2} \neq 0$ is smaller than the cases with $\lambda_{1}=0$ or $\lambda_{2}=0$, and the minimum is in the case of $\lambda_{1}=$ $\lambda_{2}=0.5$ (corresponding to Table 5). This indicates the model considering the distance and road characteristics comprehensively can provide superior distribution paths to the model considering the distance or road characteristics only.

\section{Conclusions and Future Work}

In this paper, the logistics distribution path planning in an urban area is investigated. A mathematical model considering the road characteristics and distance synthetically is proposed. In the model, the road characteristics evaluation value is defined to quantify the road characteristics. In order to obtain the road characteristics evaluation value, road characteristics evaluation index system is established and fuzzy comprehensive evaluation method is used. 
Furthermore, AGA with a customized crossover operator, in which the position of crossover point is selected according to the vehicle path in the chromosome, is designed to solve the model.

A real example is selected, in which the road characteristics evaluation is conducted, and the simulation is carried out. The results illustrate the proposed model achieves the optimal path for logistics distribution path planning in the urban area and the designed AGA can obtain the better solution and convergence speed than GA. The comparisons from results of different weight coefficients for the road characteristics and distance in the model show considering the road characteristics and distance comprehensively can produce superior distribution paths to considering the road distance or road characteristics only.

The study of this paper is helpful for the logistics distribution companies to obtain the optimal logistics distribution path in the urban area, especially in mountain cities with the roads of the characteristics of large longitudinal slopes and small flat curve corners being common.

This paper took the related factors of road characteristics into account in the model for urban regional logistics distribution path planning. However, the road characteristics factors include many aspects and categories, some of which have not been considered further, such as the road traffic state and number of lanes. In future works, these factors can be taken into account in the model. In addition, the logistics vehicle distribution model considering road characteristics can be expanded to other variants of VRP, such as VRP with multidistribution centers and more distribution stations.

\section{Data Availability}

The data used to support the conclusions of this paper are included in this study and are available upon request from the authors.

\section{Conflicts of Interest}

The authors declare that there are no conflicts of interest.

\section{Acknowledgments}

This research was funded by the National Natural Science Foundation of China under grant no. 71801144, Key Research and Department Project of Shandong Province under grant no. 2019GGX101008, and China Postdoctoral Science Foundation funded project under grant no. 2019M652437.

\section{References}

[1] S. Anily and J. Bramel, "Vehicle routing and the supply chain management," Quantitative Models For Supply Chain Management, International Series in Operations Research \& Management Science, Springer, vol. 17, pp. 148-196, Bostan, MA, USA, 1999.

[2] R. H. Ballou, Business Logistics Management: Planning, Organizing, and Controlling the Supply Chain, Prentice-Hall, Upper Saddle River, NJ, USA, 2004.
[3] D. Cattaruzza, N. Absi, D. Feillet, and J. González-Feliu, "Vehicle routing problems for city logistics," EURO Journal on Transportation and Logistics, vol. 6, no. 1, pp. 51-79, 2017.

[4] B. Chen, R. Qu, R. Bai, and W. Laesanklang, "A hyperheuristic with two guidance indicators for bi-objective mixedshift vehicle routing problem with time windows," Applied Intelligence, vol. 48, no. 12, pp. 4937-4959, 2018.

[5] E. B. Alaia, I. H. Dridi, H. Bouchriha, and P. Borne, "Genetic algorithm for multi-criteria optimization of multi-depots pick-up and delivery problems with time windows and multivehicles," Acta Polytechnica Hungarica, vol. 12, no. 8, pp. 155-174, 2015.

[6] H. C. Lu, Y. W. Yang, and L. T. Su, "Ant colony optimization solutions for logistic route planning with pick-up and delivery," in Proceedings of the 2016 IEEE International Conference on Systems, Man, and Cybernetics, pp. 808-813, Budapest, Hungary, October 2016.

[7] A. M. A. Soliman, "Effect of road roughness on the vehicle ride comfort and rolling resistance," in Proceedings of the 2006 SAE World Congress, Detroit, MI, USA, April 2006.

[8] A. Louhghalam, M. Akbarian, and F.-J. Ulm, "Scaling relationships of dissipation-induced pavement-vehicle interactions," Transportation Research Record: Journal of the Transportation Research Board, vol. 2457, no. 1, pp. 95-104, 2014.

[9] C. Wang, M. A. Quddus, and S. G. Ison, "The effect of traffic and road characteristics on road safety: a review and future research direction," Safety Science, vol. 57, pp. 264-275, 2013.

[10] J. Xu, X. Wang, C. Wang, Y. M. Shao, and Z. Y. Ma, "Foot manoeuvres and workload of driver on mountainous roads with longitudinal slopes," China Journal of Highway and Transport, vol. 31, no. 1, pp. 91-100, 2018.

[11] M. Nadji, C. H. Lamarque, and M. Gothié, "Road geometry effect on traffic safety: vehicle/infrastructure modelling," in Proceedings of the 2007 International Conference on Advances in Vehicle Control and Safety, pp. 49-54, Buenos Aires, Argentina, February 2007.

[12] K. Chatti and I. Zaabar, "Estimating the effects of pavement condition on vehicle operating costs," Transportation Research Board of the National Academies, vol. 2455, 2012.

[13] G.-P. Gwon, W.-S. Hur, S.-W. Kim, and S.-W. Seo, "Generation of a precise and efficient lane-level road map for intelligent vehicle systems," IEEE Transactions on Vehicular Technology, vol. 66, no. 6, pp. 4517-4533, 2017.

[14] J. Mahdi, J. Ehsan, Y. L. Gu, and K. Shunsuke, "Towards highdefinition 3D urban mapping: road feature-based registration of mobile mapping systems and aerial imagery," Remote Sensing, vol. 9, no. 10, pp. 975-1005, 2017.

[15] A. Mohamed, M. M. M. Fouad, E. Elhariri et al., "RoadMonitor: an intelligent road surface condition monitoring system," Advances in Intelligent Systems and Computing, vol. 323, pp. 377-387, 2015.

[16] M. Strutu, G. Stamatescu, and D. Popescu, "A mobile sensor network based road surface monitoring system," in Proceedings of the 17th International Conference On System Theory, Control and Computing, pp. 630-634, Sinaia, Romania, October 2013.

[17] G. B. Dantzig and J. H. Ramser, "The truck dispatching problem," Management Science, vol. 6, no. 1, pp. 80-91, 1959.

[18] S. Raff, L. D. Bodin, B. L. Golden, A. A. Assad, and M. O. Ball, "Routing and scheduling of vehicles and crews: the state of the art," Computers \& Operations Research, vol. 10, no. 2, pp. 63-211, 1983. 
[19] M. Desrochers, J. Desrosiers, and M. Solomon, “A new optimization algorithm for the vehicle routing problem with time windows," Operations Research, vol. 40, no. 2, pp. 342354, 1992.

[20] J. Desrosiers, Y. Dumas, M. M. Solomon, and F. Soumis, "Chapter 2 Time constrained routing and scheduling," Handbooks in Operations Research and Management Science, vol. 8, pp. 35-139, 1995.

[21] M. L. Fisher, K. O. Jörnsten, and O. B. G. Madsen, "Vehicle routing with time windows: two optimization algorithms," Operations Research, vol. 45, no. 3, pp. 488-492, 1997.

[22] D. Pisinger and S. Ropke, "A general heuristic for vehicle routing problems," Computers \& Operations Research, vol. 34, no. 8, pp. 2403-2435, 2007.

[23] Z. C. Wang, M. X. Zhou, J. Li, and J. Fan, "Research in capacitated vehicle routing problem based on modified hybrid particle swarm optimization," in Proceedings of the International Conference on Intelligent Computing and Intelligent Systems, pp. 289-293, IEEE, Shanghai, China, November 2009.

[24] H. C. W. Lau, T. M. Chan, W. T. Tsui, and W. K. Pang, "Application of genetic algorithms to solve the multidepot vehicle routing problem," IEEE Transactions On Automation Science And Engineering, vol. 7, no. 2, pp. 383-392, 2010.

[25] Y. Wang, X. Ma, Z. Li, Y. Liu, M. Xu, and Y. Wang, "Profit distribution in collaborative multiple centers vehicle routing problem," Journal of Cleaner Production, vol. 144, pp. 203219, 2017.

[26] M. A. Mohammed, M. S. Ahmad, and S. A. Mostafa, "Using genetic algorithm in implementing capacitated vehicle routing problem," in Proceedings of the International Conference on Computer and Information Science, pp. 257-261, Kuala Lumpur, Malaysia, June 2012.

[27] T. Vidal, T. G. Crainic, M. Gendreau, and C. Prins, "A hybrid genetic algorithm with adaptive diversity management for a large class of vehicle routing problems with time-windows," Computers \& Operations Research, vol. 40, no. 1, pp. 475-489, 2013.

[28] L. L. Liao, X. S. Cai, H. D. Huang, and Y. H. Liu, "Vehicle routing with time windows based on two-stage optimization algorithm," in Proceedings of the 28th Chinese Control and Decision Conference, pp. 4741-4745, Yinchuan, China, May 2016.

[29] M. A. Mohammed, M. K. Abd Ghani, R. I. Hamed, S. A. Mostafa, M. S. Ahmad, and D. A. Ibrahim, "Solving vehicle routing problem by using improved genetic algorithm for optimal solution," Journal of Computational Science, vol. 21, pp. 255-262, 2017.

[30] R. Liu and Z. Jiang, "A hybrid large-neighborhood search algorithm for the cumulative capacitated vehicle routing problem with time-window constraints," Applied Soft Computing, vol. 80, pp. 18-30, 2019.

[31] H. Lei, G. Laporte, and B. Guo, "The capacitated vehicle routing problem with stochastic demands and time windows," Computers \& Operations Research, vol. 38, no. 12, pp. 17751783, 2011.

[32] Y. Wang, K. Assogba, J. Fan, M. Xu, Y. Liu, and H. Wang, "Multi-depot green vehicle routing problem with shared transportation resource: integration of time-dependent speed and piecewise penalty cost," Journal of Cleaner Production, vol. 232, pp. 12-29, 2019.

[33] G. H. Wang and W. L. Bian, "Vehicle routing problem with simultaneous delivery and pick-up under different weights," in Proceedings of the International Conference on Logistics, Informatics and Service Sciences, Sydney, Australia, July 2016.

[34] E. E. Zachariadis, C. D. Tarantilis, and C. T. Kiranoudis, "Vehicle routing strategies for pick-up and delivery service under two dimensional loading constraints," Operational Research, vol. 17, no. 1, pp. 115-143, 2017.

[35] P. Sitek and J. Wikarek, "Capacitated vehicle routing problem with pick-up and alternative delivery (CVRPPAD): model and implementation using hybrid approach," Annals of Operations Research, vol. 273, no. 1-2, pp. 257-277, 2019.

[36] Y. Shi, T. Boudouh, and O. Grunder, "An efficient tabu search based procedure for simultaneous delivery and pick-up problem with time window," IFAC-PapersOnLine, vol. 51, no. 11, pp. 241-246, 2018.

[37] C. Lagos, G. Guerrero, E. Cabrera, A. Moltedo, F. Johnson, and F. Paredes, "An improved particle swarm optimization algorithm for the VRP with simultaneous pickup and delivery and time windows," IEEE Latin America Transactions, vol. 16, no. 6, pp. 1732-1740, 2018.

[38] C. Wang, D. Mu, F. Zhao, and J. W. Sutherland, "A parallel simulated annealing method for the vehicle routing problem with simultaneous pickup-delivery and time windows," Computers \& Industrial Engineering, vol. 83, pp. 111-122, 2015.

[39] H.-F. Wang and Y.-Y. Chen, "A genetic algorithm for the simultaneous delivery and pickup problems with time window," Computers \& Industrial Engineering, vol. 62, no. 1, pp. 84-95, 2012.

[40] K. Saâdia, EI. Y. Othmane, B. Chakib, and B. Yahya, "A genetic algorithm for solving a multi-trip vehicle routing problem with time windows and simultaneous pick-up and delivery in a hospital complex," in Proceedings of the $3 \mathrm{rd}$ International Conference On Machine Learning and Soft Computing, pp. 76-81, Da Lat, Vietnam, January 2019.

[41] C. L. Ma, Y. Y. Yang, L. H. Wang, C. Chu, C. Ma, and L. H. An, "Research on distribution route with time window and onboard constraint based on tabu search algorithm," EURASIP Journal on Wireless Communications and Networking, vol. 2019, no. 1, 2019.

[42] M. Cherkesly, G. Desaulniers, and G. Laporte, “A populationbased metaheuristic for the pickup and delivery problem with time windows and LIFO loading," Computers \& Operations Research, vol. 62, pp. 23-35, 2015.

[43] C. X. Ren, H. Wang, C. C. Yin, and F. S. Liu, "Study on the logistics distribution route problem based on road traffic volume," in Proceedings of the International Conference of Logistics Engineering and Management, Chengdu, China, October 2010.

[44] R. Kurniawan, M. D. Sulistiyo, and G. S. Wulandari, "Genetic algorithm for capacitated vehicle routing problem with considering traffic density," in Proceedings of the International Conference on Information Technology Systems and Innovation, Bandung Bali, Indonesia, November 2015.

[45] J. Milton and F. Mannering, "The relationship among highway geometrics, traffic-related elements and motor-vehicle accident frequencies," Transportation, vol. 25, no. 4, pp. 395-413, 1998.

[46] R. Haynes, I. R. Lake, S. Kingham, C. E. Sabel, J. Pearce, and R. Barnett, "The influence of road curvature on fatal crashes in New Zealand," Accident Analysis \& Prevention, vol. 40, no. 3, pp. 843-850, 2008.

[47] C. Wang, M. Quddus, and S. Ison, "The effects of area-wide road speed and curvature on traffic casualties in England," 
Journal of Transport Geography, vol. 17, no. 5, pp. 385-395, 2009.

[48] M. Ross, "Automobile fuel consumption and emissions: effects of vehicle and driving characteristics," Annual Review of Energy and the Environment, vol. 19, no. 1, pp. 75-112, 1994.

[49] W. Yong, X. Guan, B. Wang, and M. Ding, "Research on the real-time identification approach of longitudinal road slope and maximum road friction coefficient," International Journal of Vehicle Design, vol. 79, no. 1, pp. 18-42, 2019.

[50] P. L. Fatty, "The decision making for leaders: the analytical hierarchy process for decisions in a complex world," European Journal of Operational Research, vol. 42, no. 1, pp. 107-109.

[51] X. D. Wang and X. Y. Peng, "Application of analytic hierarchy process in evaluating education equipment efficiency factors," in Proceedings of the International Conference on Business Management and Electronic Information, pp. 96-100, Guangzhou, China, May 2011.

[52] D. Ergu, G. Kou, Y. Shi, and Y. Shi, "Analytic network process in risk assessment and decision analysis," Computers \& Operations Research, vol. 42, pp. 58-74, 2014.

[53] Y. Wang and Z. Y. Gou, "Road safety evaluation based on fuzzy logic," Journal of Tongji University, vol. 36, no. 1, pp. 47-51, 2008.

[54] S. Shao, A. L. Huang, and H. J. Liu, "City freight intelligent scheduling model based on genetic algorithm," in Proceedings of the 28th International Conference on Software Engineering and Knowledge Engineering, pp. 297-300, Redwood City, CA, USA, July 2016.

[55] S. Karakatič and V. Podgorelec, "A survey of genetic algorithms for solving multi-depot vehicle routing problem," Applied Soft Computing Journal, vol. 27, pp. 519-532, 2015.

[56] Q. Li, S. J. Xie, X. H. Tong, and Z. L. Wang, "Self-adaptive genetic algorithm for the shortest path planning of vehicles and its comparison with Dijkstra and A*algorithms," Journal of University of Science and Technology Beijing, vol. 28, no. 11, pp. 1082-1086, 2006.

[57] W. Wu and Q. Q. Ruan, "A gene-constrained genetic algorithm for solving short path problem," in Proceedings of the 7th International Conference on Signal Processing, pp. 25102513, Beijing, China, August 2004.

[58] Z. Y. Sun, Z. L. Guan, and Q. Wang, "An improved adaptive genetic algorithm for vehicle routing problem," in Proceedings of the International Conference on Logistics Systems and Intelligent Management, pp. 116-120, Harbin, China, January 2010.

[59] M. Srinivas and L. M. Patnaik, "Adaptive probabilities of crossover and mutation in genetic algorithms," IEEE Transactions on Systems, Man, and Cybernetics, vol. 24, no. 4, pp. 656-667, 1994.

[60] G. Gao, Z. Wang, X. Liu, and T. Li, “An empirical spatial accessibility analysis of Qingdao city based on multisource data," Journal of Advanced Transportation, vol. 2020, Article ID 7570148, 19 pages, 2020.

[61] Z. Gao, J. Wang, X. Zhang, Q. Li, W. Wang, and J. Zhang, "Traffic oscillations mitigation in vehicle platoon using a carfollowing control model for connected and autonomous vehicle," Journal of Advanced Transportation, vol. 2019, Article ID 6383097, 18 pages, 2019.

[62] Z. Wang, G. Gao, X. Liu, and W. Lyu, "Verification and analysis of traffic evaluation indicators in urban transportation system planning based on multi-source data-a case study of Qingdao city, China," IEEE Access, vol. 7, Article ID 110103, 2019. 